\title{
2. Berliner Symposium des BfDI zur Informationsfreiheit
}

Anlässlich des 2. Berliner Symposiums zur Informationsfreiheit trafen sich am 6. und 7. September 2012 Abgeordnete, Verwaltungsrichter aller drei Instanzen, Informationsfreiheitsbeauftragte der Länder, Hochschullehrer, Anwender des IFG aus Ministerien und Bundesbehörden, Vertreter von Kirchen und Nichtregierungsorganisationen sowie Repräsentanten der Netzgemeinde und der Medien. Die Zahl von rund einhundert Teilnehmern belegt das stetig wachsende Interesse am Querschnittsthema „Informationsfreiheit" auf Bundes- und Landesebene.

Das Hamburgische Transparenzgesetz war - kaum im Gesetzblatt veröffentlicht - das erste große, von meinem Kollegen Prof. Johannes Caspar vorgestellte, Thema des Symposiums. Mit diesem Gesetz, das insbesondere die proaktive Veröffentlichung von Verwaltungsinformationen sehr deutlich stärkt, spielt Hamburg, sportlich formuliert, im Spitzenfeld der ersten Bundesliga. Eine vergleichbare Regelung im Informationsfreiheitsgesetz des Bundes würde ich sehr begrüßen.

Prof. Ingolf Pernice von der Humboldt-Universität Berlin lenkte den Blick auf die europarechtlichen Wurzeln und die Entwicklung des Umweltinformationsrechts. Mit dem Umweltinformationsrecht habe der Paradigmenwechsel vom arkanen zum offenen Staat eingesetzt. Dieser Paradigmenwechsel könne bei der Interpretation des Artikel 5 Abs. 1 Satz 1, 2. Halbsatz des Grundgesetzes nicht länger außer Betracht bleiben.

Den zweiten Tag des Symposiums eröffnete Prof. Friedrich Schoch (Albert-Ludwigs-Universität Freiburg) mit seiner Analyse und pointierten Kritik der nationalen und der EuGH-Rechtsprechung im Spannungsfeld von Informationsfreiheit und Datenschutz. Prof. Schoch sprach sich auch für eine Ausdehnung meiner Beratungs-, Kontroll- und Ombudsaufgaben auf das Umweltinformationsgesetz und das Verbraucherinformationsgesetz aus.

Dr. Angela Spelsberg und Dieter Hüsgen (Transparency Deutschland) schilderten in ihrer Fallstudie die (Prozess-)Geschichte ihres IFG-Antrags bei der Kassenärztlichen Bundesvereinigung. Angesichts der Knappheit der Gesundheitskassen und der Notwendigkeit transparenter und rationaler Verwendung der Beiträge der Versicherten ist dies ein unbedingt auch mit den Mitteln des Informationsfreiheitsgesetzes weiter auszuleuchtendes Feld. Die im Wesentlichen stattgebende Entscheidung des Verwaltungsgerichtes Berlin vom 1. Juni 2012 (Az. VG 2 K 177.11) ist deshalb auch aus meiner Sicht ein weiterer informationsfreiheitsrechtlicher Meilenstein.

Nachdem Dr. Elisabeth Musch vom Deutschen Forschungsinstitut für öffentliche Verwaltung Speyer im vergangenen Jahr beim
1. Berliner Symposium das „Design“ der vom Innenausschuss des Deutschen Bundestages in Auftrag gegebenen Evaluation erläutert hatte, konnte sie jetzt das Ergebnis der im letzten Frühsommer aufgenommenen Evaluation gemeinsam mit ihrem Kollegen Dr. Alfred Debus vorstellen.

Der Justiziar des Bundeskartellamtes Jörg Nothdurft setzte sich in seinem Impulsreferat, pointiert mit anschaulicher und zugleich differenzierter Argumentation für den Schutz von Betriebs- und Geschäftsgeheimnissen im Kontext des Informationszuganges beim Bundeskartellamt ein.

Dr. Helene Groß aus dem Bundesministerium des Innern gab einen Überblick über den Stand des Open Government Projektes der Bundesregierung und der Zusammenarbeit mit den Ländern. Anfang 2013 soll der Prototyp eines ebenenübergreifenden Open-Data-Portals online gehen. Die Referentin verwies auch auf die im Auftrag des Bundesministeriums des Innern verfasste und veröffentlichte Studie „Open Government Data Deutschland“.

Die nationale Perspektive wurde schließlich von einer europäischen abgelöst: Dr. Carl-Christian Buhr von der Europäischen Kommission referierte zur Funktion der öffentlichen Information als Wirtschaftsgut. Eines der zentralen Anliegen der Kommission sei die Stärkung der Verfügbarkeit und Weiterverwendung von Daten, wozu neben der Datenportabilität auch die Gewährleistung eines hohen Datenschutzniveaus durch die geplante EU-Datenschutzverordnung gehöre.

Mein Fazit des 2. Symposiums: Bei einzelnen Bundesländern sehe ich eine sehr gute normative und technische Umsetzung des Open-Data-Gedankens; zugleich gibt es immer noch Bundesländer ohne Informationsfreiheitsgesetz. Auf Bundesebene ist insbesondere das proaktive Element endlich weiter zu entwickeln. Das Hamburgische Transparenzgesetz, aber auch der Entwurf eines neuen Berliner Transparenz- und Informationsfreiheitsgesetzes der Fraktion Bündnis 90/Die Grünen sollten hier als Vorbild genommen werden.

Abschließend möchte ich diese Gelegenheit auch nutzen, Sie auf die vom 18. bis 20. September 2013 von meinem Berliner Kollegen Alexander Dix und mir gemeinsam auszurichtende Internationale Konferenz der Informationsfreiheitsbeauftragten (ICIC) aufmerksam zu machen. Die Beiträge des Symposiums sowie Informationen zur Internationalen Konferenz finden Sie unter Informationsfreiheit.bund.de.

Peter Schaar

Der Bundesbeauftragte für den Datenschutz und die Informationsfreiheit 